\title{
Paroxysmal ventricular tachycardia showing fusion with reciprocal ventricular beats
}

\author{
Aart Vermeulen and Hein J. Wellens \\ From the University Department of Cardiology and Clinical Physiology, Wilhelmina Gasthuis, \\ Amsterdam, The Netherlands
}

A case is described of ventricular tachycardia with 2 to I retrograde ventriculo-atrial conduction, ventricular reciprocal beats, and fusion between the latter and the ectopic ventricular focus.

The use of oesophageal and intra-atrial leads during ventricular tachycardia has shown that retrograde ventriculo-atrial conduction is common (Kistin, I96I). Comparable to antegrade conduction, retrograde conduction through the atrioventricular (AV) junction can show first, second, and third degree heart block and Wenckebach type of conduction delay. We know that impaired $\mathrm{AV}$ and ventriculo-atrial conduction and the presence of differences in conduction velocity and refractoriness of different parts of the AV junction favour the development of reciprocal beating (Moe and Mendez, 1966).

The simultaneous occurrence of ventricular tachycardia and reciprocal ventricular beating is usually prevented by the discharge rate of the ectopic ventricular focus creating refractoriness of the myocardium for the descending reciprocal impulse.

Recently we saw a man who had a ventricular tachycardia with irregular interventricular intervals and ventriculo-atrial conduction. In this patient fortuitously timed reciprocal ventricular beats resulted in fusion with the ectopic ventricular complex.

\section{Case report}

The tracing was recorded from a 78-year-old man who was admitted to our department for evaluation of intractable attacks of tachycardia. He was treated with $300 \mathrm{mg}$. quinidine 6 times daily at the time the electrocardiogram was registered. Leads I, II, III, VI, and V6 were recorded simultaneously at a paper speed of $25 \mathrm{~mm}$./sec.

Fig. IA shows a paroxysmal tachycardia, frequency approximately II5 a minute. The QRS complexes have a width of 0.18 sec. and a complete right bundle-branch block configuration with initial negativity in lead Vr. The intervals

between the QRS complexes are irregular, possibly due to an exit block of the ectopic pacemaker, the latter probably aggravated by the

FIG. I (A) Leads $I, I I, I I I, V_{I}$, and V6 during a run of irregular ventricular tachycardia ending with a small $Q R S$ complex marked by an arrow, representing a reciprocal ventricular beat (for explanation see text). In the diagram three levels are shown: atrium, $A V$ junction, and ventricle. The numbers (expressed in msec.) correspond with the interatrial interval, ventriculo-atrial conductiontime, and interventricular interval.
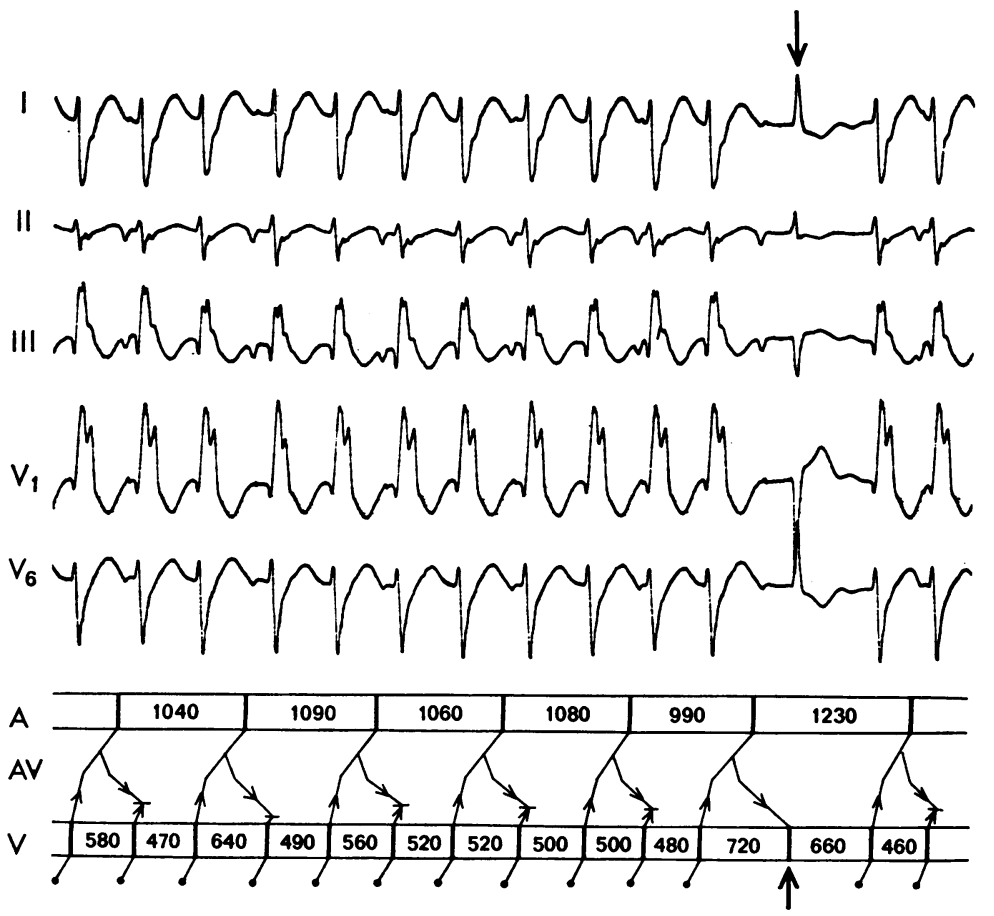
quinidine medication. As shown in the diagram, during the tachycardia every second QRS complex is followed by a $\mathbf{P}$ wave. The configuration of this $\mathbf{P}$ wave points to atrial activation in a caudo-cranial direction. The $Q P$ intervals vary inversely with the RR intervals; in other words, a long RR interval is followed by a short QP interval and vice versa.

The sequence of $Q R S$ complexes and $P$ waves suggests 2 to I retrograde ventriculo-atrial conduction during the tachycardia. At the arrow during a pause in the tachycardia, a normally propagated beat follows $0.28 \mathrm{sec}$. after a retrograde $P$ wave. This phenomenon was repeatedly noticed and was only seen when a retrograde $P$ wave followed the last QRS complex of the tachycardia. The interval between the last QRS complex of the tachycardia and the small QRS complex following it ranged from 680 to $720 \mathrm{msec}$. This makes it unlikely that we are dealing with coupled beats from an ectopic focus. An AV junctional parasytole is also unlikely, as we could not show a common denominator in the distance between the smaller QRS complexes. Longer pauses during the tachycardia without retrograde $\mathbf{P}$ waves were terminated by a new paroxysmal tachycardia, the first complex having already a QRS width of $0.18 \mathrm{sec}$. This latter finding makes an AV junctional escape beat unlikely in the explanation of the small QRS complex and favours reciprocal ventricular beating as the causal mechanism.

The beginning of the tachycardia after a long pause with a QRS complex of identical contour and width $(0.18 \mathrm{sec}$.) as the following complex of the tachycardia suggests a ventricular origin of the tachycardia. Fig. IB shows during the tachycardia two complexes (arrows) which differ in configuration as compared to the tachycardia complexes. Both complexes terminate a relatively long interventricular interval and are preceded by retrograde $\mathbf{P}$ waves. In our opinion they represent fusion beats between a reciprocal ventricular beat, as shown in Fig. IA, and a complex from the ventricular focus. Fig. 2 shows the electrocardiogram taken after the patient reverted to sinus rhythm.

\section{Discussion}

Fusion beats during a ventricular tachycardia cannot only result from antegrade conduction through the AV junction or from another ectopic ventricular focus, but also from reciprocal ventricular beating. It is obvious that a relatively slow and irregular rate $(100-130$ beats per minute) of the ventricular tachycardia facilitates the occurrence and recognition of this phenomenon. The occurrence of fusion beats during ventricular tachycardia due to reciprocal ventricular beats was first described by Malinow and Langendorf (1948). Other cases were presented by Kistin (I96r) and Latour, Puech, and Hertault (1962). The experimental proof for the occurrence of this phenomenon and the dependence

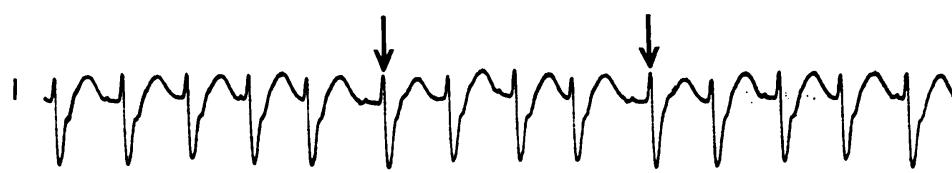

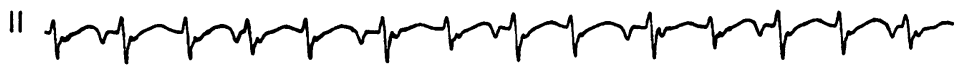

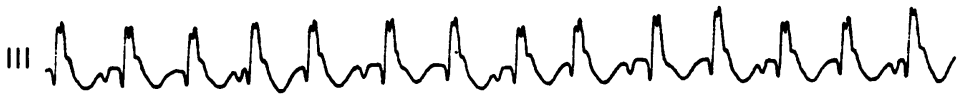


FIG. I (B) Same leads as in Fig. IA. The two beats marked by arrows represent fusion beats between reciprocal ventricular beats and ventricular beats originating from the ventricular focus responsible for the ventricular tachycardia.

FIG. 2 The I4-lead electrocardiogram during normal sinus rhythm.

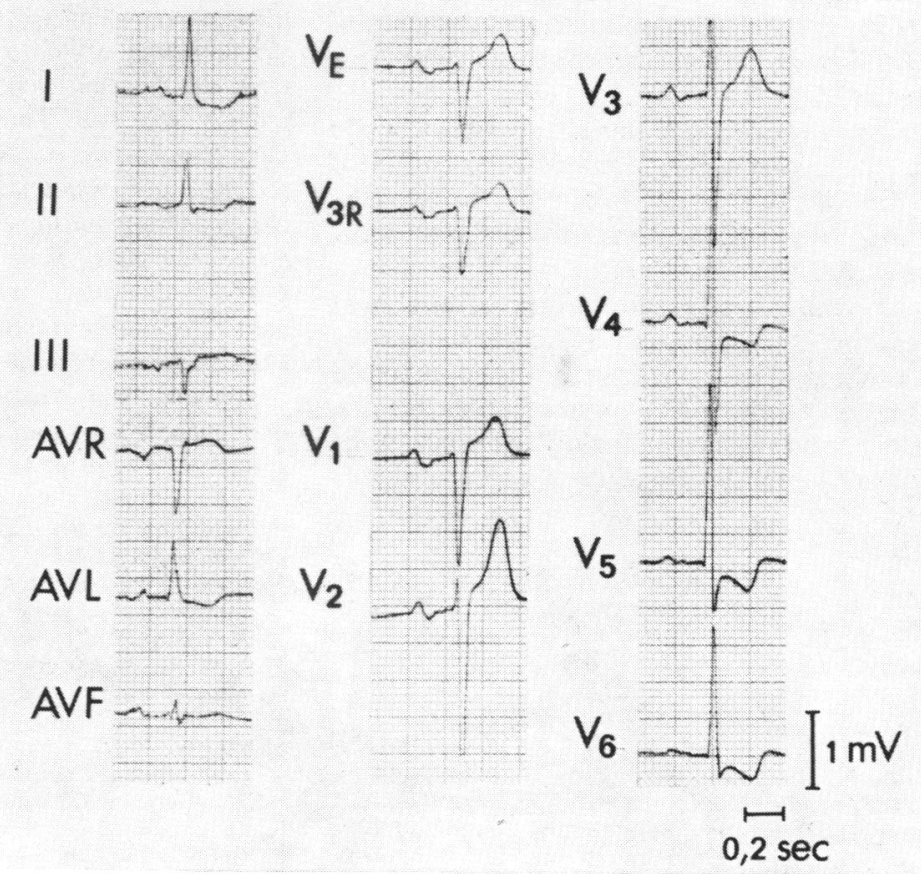


upon a critical ventricular rate was shown by Slama, Cachin, and Bourthoumieux (1965) by stimulating the ventricle at different rates with the help of a pacemaker. The 2 : I relation between the! QRS complexes and the retrograde atrial activation in our patient requires further explanation. One could simply say that the refractory period of the AV junction is prolonged. This would cause the retrograde impulse resulting from every second ventricular complex to find the AV junction still refractory from the first impulse. If one believes, however, that longitudinal dissociation of the AV junction is the basis for the occurrence of reciprocal beating, the 2: I ventriculo-atrial conduction can be explained in a more attractive way. One has to assume then that in the upper half of the AV junction two pathways ( $\alpha$ and $\beta$ ) with different properties are present which converge upon a common pathway in the lower half of the AV junction (Fig. 3). During ventricular tachycardia the impulse from the ventricular focus travels upwards over the common pathway and the $\alpha$ path towards the atrium, the $\beta$ pathway being refractory. Re-entry into the AV junction takes place by way of the $\beta$ pathway. This impulse on descending into the common pathway collides upon the ascending impulse resulting from the second ectopic ventricular complex, thereby preventing retrograde atrial activation from this ventricular complex. That such a mechanism is very likely, is demonstrated by the finding that when a paroxysm of ventricular tachycardia ends with retrograde conduction to the atrium, it is followed by a reciprocal ventricular beat (Fig. IA).

As shown in Fig IA and IB, apparently only after the shortest $R R$ intervals does sufficient slowing of the impulse take place in the dissociated upper half of the AV node to complete a full reciprocal cycle.

Depending upon the timing of the next discharge of the ectopic ventricular focus only the reciprocal beat or fusion complex between this complex and the ectopic complex results. Fig. 2 shows how during normal sinus rhythm the QRS complexes were completely identical to the ones seen during reciprocal beating.

We wish to acknowledge the help and advice of Professor D. Durrer during the preparation of this report.

\section{References}

Kistin, A. D. (196I). Retrograde conduction to the atria in ventricular tachycardia. Circulation, 24, 236.

Latour, H., Puech, P., and Hertault, J. (1962). L'écho (conduction réciproque) en cours de tachycardie
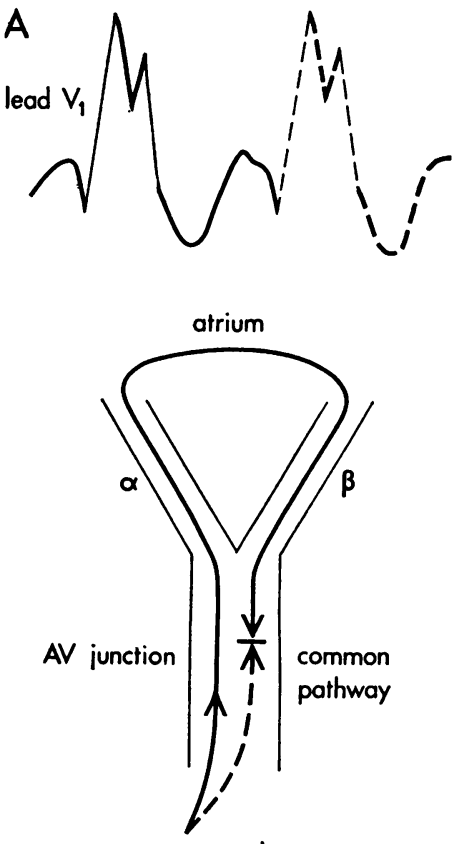

ventricle

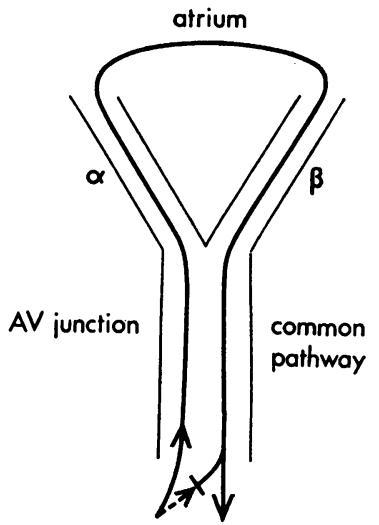

ventricle

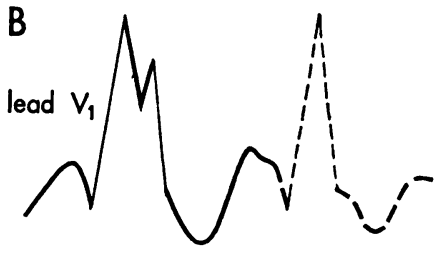

FIG. 3 Scheme explaining $(A) 2$ to I ventriculo-atrial conduction during ventricular tachycardia, and $(B)$ the occurrence of fusion between a reciprocal ventricular beat and an ectopic ventricular complex. $(A)$ After traversing the common pathway the impulse from the ectopic ventricular focus travels towards the atrium by way of the $\alpha$ path, the $\beta$ path being refractory. Re-entry into the AV junction is possible via the $\beta$ pathway. Retrograde conduction from the second ventricular complex (broken line) collides in the common pathway with the descending impulse from the first ventricular complex. This sequence leads to 2 to I ventriculo-atrial conduction. (B) When the second ectopic ventricular complex occurs later, the impulse resulting from the first ectopic ventricular complex is able to pass the common pathway leading to a reciprocal ventricular beat. Depending upon the discharge interval of the ectopic ventricular focus, a true reciprocal ventricular beat occurs, or there is fusion between a reciprocal beat and the ectopic ventricular complex (the latter is shown in this Figure).

ventriculaire. Archives des Maladies du Coeur et des Vaisseaux, 55, 180.

Malinow, M. R., and Langendorf, R. (1948). Different mechanisms of fusion beats. American Heart fournal, 35, 448.

Moe, G. K., and Mendez, C. (1966). The physiologic basis of reciprocal rhythm. Progress in Cardiovascular Diseases, 8, 46r.

Slama, R., Cachin, J. C., and Bourthoumieux, A. (1965). Le phénomène de Luciani-Wenckebach et les systoles en écho dans les conductions rétrogrades ventriculo-auriculaires. Archives des Maladies $d u$ Coeur et des Vaisseaux, 58, 648. 